\title{
Correction to: Exercise Addiction in Amateur Runners
}

\author{
Abel Nogueira López ${ }^{1}$ (D) Alfonso Salguero ${ }^{1,2} \cdot$ Olga Molinero $^{1,2} \cdot$ Antonio Rosado $^{3}$. \\ Sara Márquez ${ }^{1,2}$
}

Published online: 01 June 2021

(C) Springer Science+Business Media, LLC, part of Springer Nature 2021

\section{Correction to: International Journal of Mental Health and Addiction https://doi.org/10.1007/s11469-021-00504-3}

As a result of an error in the processing of this article during the publication process, it was necessary to re-publish this article with the following significant changes:

\section{Title}

The title of the initial version is Exercise addiction in amateur runners, it was changed to Exercise addiction in competitive amateur runners.

\begin{abstract}
It was completely modified in line with the new methodology applied, in addition to minor terminological changes (ex. "gender" by "sex").

\section{Introduction}

Grammatical and translation modifications were made, some terms on the research question were adapted, some of the concepts included in this section was expanded and improved. and two paragraphs on previous empirical research on the research question were added.
\end{abstract}

\section{Method}

\section{Participants}

Further data (sociodemographic and athletic) on the sample analysed was provided.

The online version of the original article can be found at https://oi.org/10.1007/s11469-021-00504-3

Abel Nogueira López

abel.nogueira@unini.org; anogul00@estudiantes.unileon.es

1 Institute of Biomedicine (IBIOMED), University of León, León, Spain

2 Department of Physical Education and Sport Sciences, University of León, León, Spain

3 Faculty of Human Kinetics, University of Lisbon, Lisbon, Portugal 
Table 1 Main characteristics of the sample (frequency, percentage, mean and standard deviation)

\begin{tabular}{|c|c|c|c|c|c|c|c|c|c|c|c|c|c|}
\hline & & \multicolumn{2}{|l|}{ Min } & \multicolumn{2}{|l|}{ Low } & \multicolumn{2}{|c|}{ High } & \multicolumn{2}{|c|}{ Max } & \multicolumn{4}{|c|}{ Total } \\
\hline & & $\mathrm{N}$ & $\%$ & $\mathrm{~N}$ & $\%$ & $\mathrm{~N}$ & $\%$ & $\mathrm{~N}$ & $\%$ & $\mathrm{~N}$ & $\%$ & Mean & SD \\
\hline \multirow[t]{2}{*}{ Sex } & Men & 149 & 76.8 & 134 & 78.8 & 84 & 82.4 & 40 & 83.3 & 407 & 79.2 & & \\
\hline & Women & 45 & 23.2 & 36 & 21.2 & 18 & 17.6 & 8 & 16.7 & 107 & 20.8 & & \\
\hline \multirow[t]{3}{*}{ Age } & $\leq 36$ & 86 & 44.3 & 55 & 32.4 & 30 & 29.4 & 14 & 29.2 & 185 & 36.0 & 38.29 & 8.75 \\
\hline & $36-55$ & 98 & 50.5 & 109 & 64.1 & 68 & 66.7 & 32 & 66.7 & 307 & 59.7 & & \\
\hline & $\geq 56$ & 10 & 5.2 & 6 & 3.5 & 4 & 3.9 & 2 & 4.2 & 22 & 4.3 & & \\
\hline \multirow[t]{2}{*}{ Club-membership } & $\bar{Y}$ es & 85 & 43.8 & 90 & 52.9 & 50 & 49.0 & 34 & 70.8 & 259 & 50.4 & & \\
\hline & No & 109 & 56.2 & 80 & 47.1 & 52 & 51.0 & 14 & 29.2 & 255 & 49.6 & & \\
\hline \multirow[t]{4}{*}{ Civil Status } & Single & 88 & 45.4 & 67 & 39.4 & 33 & 32.4 & 17 & 35.4 & 205 & 39.9 & & \\
\hline & Married & 84 & 43.3 & 83 & 48.8 & 51 & 50.0 & 23 & 47.9 & 241 & 46.9 & & \\
\hline & Divorced & 8 & 4.1 & 4 & 2.4 & 6 & 5.9 & 3 & 6.3 & 21 & 4.1 & & \\
\hline & $\begin{array}{l}\text { Common Law } \\
\text { Partner }\end{array}$ & 14 & 7.2 & 16 & 9.4 & 12 & 11.8 & 5 & 10.4 & 47 & 9.1 & & \\
\hline \multirow[t]{2}{*}{ Parenthood } & Yes & 84 & 43.3 & 85 & 50 & 54 & 52.9 & 23 & 47.9 & 246 & 47.9 & & \\
\hline & No & 110 & 56.7 & 85 & 50 & 48 & 47.1 & 25 & 52.1 & 268 & 52.1 & & \\
\hline \multirow{4}{*}{$\begin{array}{c}\text { Employment } \\
\text { Situation }\end{array}$} & Study & 25 & 12.9 & 17 & 10 & 5 & 4.9 & 3 & 6.3 & 50 & 9.7 & & \\
\hline & Employed & 153 & 78.9 & 146 & 85.9 & 91 & 89.2 & 39 & 81.3 & 429 & 83.5 & & \\
\hline & Unemployed & 12 & 6.2 & 5 & 2.9 & 4 & 3.9 & 5 & 10.4 & 26 & 5.1 & & \\
\hline & Retired & 4 & 2.1 & 2 & 1.2 & 2 & 2.0 & 1 & 2.1 & 9 & 1.8 & & \\
\hline \multirow{5}{*}{$\begin{array}{l}\text { Reasons to start } \\
\text { running }\end{array}$} & Healthy & 134 & 69.1 & 134 & 78.8 & 77 & 75.7 & 37 & 77.1 & 382 & 74.3 & & \\
\hline & Cheap Sport & 37 & 19.1 & 23 & 13.5 & 12 & 11.8 & 6 & 12.5 & 78 & 15.2 & & \\
\hline & Social & 3 & 1.5 & 1 & 0.6 & 1 & 1.0 & & & 5 & 1.0 & & \\
\hline & Personal Goals & 3 & 1.5 & 1 & 0.6 & 3 & 2.9 & 4 & 8.3 & 11 & 7.4 & & \\
\hline & Others & 17 & 8.8 & 11 & 6.5 & 9 & 8.8 & 1 & 2.1 & 38 & 2.1 & & \\
\hline \multirow{3}{*}{$\begin{array}{l}\text { Running expertise } \\
\text { years }\end{array}$} & $<5$ years & 92 & 47.4 & 59 & 34.7 & 45 & 44.1 & 13 & 27.1 & 209 & 40.7 & & \\
\hline & $\begin{array}{c}\text { Between } 5 \text { and } \\
10 \text { years }\end{array}$ & 40 & 20.6 & 56 & 32.9 & 27 & 26.5 & 19 & 39.6 & 142 & 27.6 & & \\
\hline & More 10 years & 62 & 32.0 & 55 & 32.4 & 30 & 29.4 & 16 & 33.3 & 163 & 31.7 & & \\
\hline \multirow{3}{*}{$\begin{array}{l}\text { Duration of training } \\
\text { session }\end{array}$} & $<60$ & 73 & 37.6 & 72 & 42.4 & 18 & 17.6 & 10 & 20.8 & 135 & 26.3 & & \\
\hline & $60-90$ & 116 & 59.8 & 64 & 37.6 & 79 & 77.5 & 32 & 66.7 & 356 & 69.3 & & \\
\hline & $>90$ & 5 & 2.6 & 34 & 20.0 & 5 & 4.9 & 6 & 12.5 & 23 & 4.5 & & \\
\hline \multirow[t]{3}{*}{$\mathrm{Km} /$ week } & $\leq 55$ & 160 & 82.5 & 118 & 69.4 & 66 & 64.7 & 26 & 54.2 & 370 & 73.0 & 43.31 & 22.26 \\
\hline & $\overline{56}-85$ & 31 & 16.0 & 48 & 28.2 & 29 & 28.4 & 15 & 31.3 & 123 & 23.9 & & \\
\hline & $\geq 86$ & 3 & 1.5 & 4 & 2.4 & 7 & 6.9 & 7 & 14.6 & 21 & 4.1 & & \\
\hline \multirow{3}{*}{$\begin{array}{l}\text { Training } \\
\text { frequency/week }\end{array}$} & $\leq 3$ & 117 & 60.3 & 67 & 39.4 & 27 & 26.5 & 8 & 16.7 & 219 & 42.6 & 3.89 & 1.28 \\
\hline & $4-5$ & 65 & 33.5 & 81 & 47.6 & 58 & 56.9 & 24 & 50.0 & 228 & 44.4 & & \\
\hline & $6-7$ & 12 & 6.2 & 22 & 12.9 & 17 & 16.7 & 16 & 33.3 & 67 & 13.0 & & \\
\hline Date of first race & $<5$ years & 106 & 54.7 & 81 & 47.7 & 51 & 50.0 & 19 & 39.6 & 257 & 50.0 & & \\
\hline & $\begin{array}{c}\text { Between } 5 \text { and } \\
10 \text { years }\end{array}$ & 40 & 20.6 & 43 & 25.3 & 27 & 26.5 & 18 & 37.5 & 128 & 24.9 & & \\
\hline & More 10 years & 48 & 24.7 & 46 & 27.1 & 24 & 23.5 & 11 & 22.9 & 129 & 25.1 & & \\
\hline Distance of first & $10 \mathrm{~km}$ & 162 & 83.5 & 151 & 88.9 & 87 & 85.3 & 37 & 77.1 & 437 & 85.0 & & \\
\hline race & $21 \mathrm{~km}$ & 29 & 14.9 & 18 & 10.6 & 13 & 12.7 & 10 & 20.8 & 70 & 13.6 & & \\
\hline & $42 \mathrm{~km}$ & 3 & 1.5 & 1 & .6 & 2 & 2.0 & 1 & 2.1 & 7 & 1.4 & & \\
\hline Usual Distance & $10 \mathrm{~km}$ & 122 & 62.9 & 92 & 54.1 & 50 & 49.0 & 21 & 43.8 & 285 & 55.5 & & \\
\hline & $21 \mathrm{~km}$ & 53 & 27.3 & 59 & 34.7 & 38 & 37.3 & 11 & 22.9 & 161 & 31.3 & & \\
\hline & $42 \mathrm{~km}$ & 19 & 9.8 & 19 & 11.2 & 14 & 13.7 & 16 & 33.3 & 68 & 13.2 & & \\
\hline Best $10 \mathrm{~km}$ & No answer & 12 & 6.2 & 10 & 5.9 & 3 & 2.9 & 1 & 2.1 & 20 & 3.9 & & \\
\hline & $<35^{\prime}$ & 13 & 6.7 & 6 & 3.5 & 6 & 5.9 & 6 & 12.5 & 31 & 6.0 & & \\
\hline & $35^{\prime}-39^{\prime} 59^{\prime \prime}$ & 27 & 13.9 & 46 & 27.1 & 30 & 29.4 & 10 & 20.8 & 113 & 22.0 & & \\
\hline & $>40^{\prime}$ & 142 & 73.2 & 114 & 67.1 & 63 & 61.8 & 31 & 64.6 & 350 & 68.1 & & \\
\hline Best $21 \mathrm{~km}$ & No answer & 38 & 19.6 & 24 & 14.1 & 12 & 11.8 & 5 & 10.4 & 79 & 15.4 & & \\
\hline & $<1$ h $20^{\prime}$ & 8 & 4.1 & 7 & 4.1 & 9 & 8.8 & 8 & 16.7 & 32 & 6.2 & & \\
\hline & $\begin{array}{l}1 \text { h20'- } \\
1 \text { h29'59" }\end{array}$ & 31 & 16.0 & 38 & 22.4 & 25 & 24.5 & 9 & 18.8 & 103 & 20.0 & & \\
\hline
\end{tabular}


Table 1 (continued)

\begin{tabular}{|c|c|c|c|c|c|c|c|c|c|c|c|c|}
\hline & & \multicolumn{2}{|l|}{ Min } & \multicolumn{2}{|c|}{ Low } & \multicolumn{2}{|c|}{ High } & \multicolumn{2}{|c|}{ Max } & \multicolumn{3}{|c|}{ Total } \\
\hline & & $\mathrm{N}$ & $\%$ & $\mathrm{~N}$ & $\%$ & $\mathrm{~N}$ & $\%$ & $\mathrm{~N}$ & $\%$ & $\mathrm{~N}$ & $\%$ & Mean SD \\
\hline \multirow{5}{*}{ Best $42 \mathrm{~km}$} & $>1 \mathrm{~h} 30^{\prime}$ & 117 & 60.3 & 101 & 59.4 & 56 & 54.9 & 26 & 54.2 & 300 & 58.4 & \\
\hline & No answer & 95 & 49.0 & 71 & 41.8 & 35 & 34.3 & 14 & 29.2 & 215 & 41.8 & \\
\hline & $<2 \mathrm{~h} 45^{\prime}$ & 2 & 1.0 & 4 & 2.4 & 4 & 3.9 & 5 & 10.4 & 15 & 2,9 & \\
\hline & $\begin{array}{l}2 \text { h } 45^{\prime}- \\
3 \text { h14'59', }\end{array}$ & 20 & 10.3 & 28 & 16.5 & 18 & 17.6 & 6 & 12.5 & 72 & 14.0 & \\
\hline & $>3 \mathrm{~h} 15^{\prime}$ & 77 & 39.7 & 67 & 39.4 & 45 & 44.1 & 23 & 47.9 & 212 & 41.2 & \\
\hline \multirow{2}{*}{$\begin{array}{l}\text { Additional Gym } \\
\text { Attendance }\end{array}$} & Yes & 142 & 73.2 & 129 & 75.9 & 66 & 64.7 & 40 & 83.3 & 377 & 73.3 & \\
\hline & No & 52 & 26.8 & 41 & 24.1 & 36 & 35.3 & 8 & 16.7 & 137 & 36.7 & \\
\hline
\end{tabular}

\section{Measures}

Some variables were recategorized (three categories), in order to obtain study subgroups that are more similar in size. This meant that the data had to be reinterpreted, and Table 1 was totally modified.

\section{Procedure}

More details were given in order to allow for eventual replications of the study.

Table 2 Mean rank (Md) and Kruskal-Wallis' H and significant levels of the main characteristics of the sample

\begin{tabular}{|c|c|c|c|c|}
\hline & & $\mathrm{Md}$ & $\mathrm{H}$ & $\mathrm{p}$ \\
\hline \multirow[t]{3}{*}{ Age } & $\leq 36$ & 232.92 & 9.89 & $.007 * *$ \\
\hline & $36-55$ & 273.52 & & \\
\hline & $>56$ & 240.68 & & \\
\hline \multirow[t]{2}{*}{ Club-membership } & Yes & 273.72 & 6.91 & $.009 * *$ \\
\hline & No & 241.02 & & \\
\hline \multirow[t]{3}{*}{ Running expertise years } & $<5$ years & 241.79 & 7.01 & $.030 *$ \\
\hline & Between 5 and 10 years & 282.32 & & \\
\hline & More 10 years & 256.02 & & \\
\hline \multirow[t]{3}{*}{ Duration of training session } & $<60$ & 214.85 & 20.06 & $.000 * * *$ \\
\hline & $60-90^{\prime}$ & 269.34 & & \\
\hline & $>90^{\prime}$ & 324.54 & & \\
\hline \multirow[t]{3}{*}{$\mathrm{Km} /$ week } & $<=55$ & 238.38 & 26.05 & $.000 * * *$ \\
\hline & $56-85$ & 289.20 & & \\
\hline & $>=86$ & 376.17 & & \\
\hline \multirow{3}{*}{ Training frequency/week } & $<=3$ & 206.74 & 55.28 & $.000 * * *$ \\
\hline & $4-5$ & 284.42 & & \\
\hline & $6-7$ & 331.80 & & \\
\hline \multirow[t]{3}{*}{ Usual Distance } & $10 \mathrm{~km}$ & 232.94 & 10.98 & $.004 * *$ \\
\hline & $21 \mathrm{~km}$ & 253.92 & & \\
\hline & $42 \mathrm{~km}$ & 292.91 & & \\
\hline \multirow[t]{3}{*}{ Best $10 \mathrm{~km}$} & $<35$ & 257.95 & 7.74 & $.021 *$ \\
\hline & $35^{\prime}-39^{\prime} 59^{\prime \prime}$ & 277.29 & & \\
\hline & $>40^{\prime}$ & 236.96 & & \\
\hline \multirow[t]{3}{*}{ Best $21 \mathrm{~km}$} & $<1$ h20' & 271.06 & 8.97 & $.011 *$ \\
\hline & 1 h20'- 1 h 29'59” & 228.82 & & \\
\hline & $>1 \mathrm{~h} 30^{\prime}$ & 208.63 & & \\
\hline \multirow[t]{3}{*}{ Best $42 \mathrm{~km}$} & $<2 \mathrm{~h} 45^{\prime}$ & 202.43 & 6.88 & $.032 *$ \\
\hline & 2 h45'- 3 h 14'59” & 153.38 & & \\
\hline & $>3 \mathrm{~h} 15$ & 145.14 & & \\
\hline
\end{tabular}


Table 3 Effect Size (d de Cohen) to significance variables

\begin{tabular}{llll}
\hline & Eta squared $\left(\mathrm{n}^{2}\right)$ & $\mathrm{d}$ de Cohen & Effect size \\
\hline Age & .015 & .251 & Small effect \\
Club-membership & .012 & .216 & Small effect \\
Running expertise years & .010 & .199 & No effect \\
Duration of training session & .035 & .383 & Small effect \\
Km/week & .047 & .444 & Medium effect \\
Training frequency/week & .104 & .682 & Medium effect \\
Usual Distance & .018 & .268 & Small effect \\
Best $10 \mathrm{~km}$ & .011 & .213 & Small effect \\
Best $21 \mathrm{~km}$ & .014 & .235 & Small effect \\
Best $42 \mathrm{~km}$ & .010 & .196 & No effect \\
\hline
\end{tabular}

\section{Statistical Analysis}

New analysis was carried out considering all the recommendations made, modifying the calculation of cohen's $d$ and replacing the correlations by crosstab analysis.

The structure of the analyses were modified following a logic sequence.

\section{Results}

All the section was modified considering all the recommendations and rewriting the results section, changing all tables and creating a new one (Table 5).

Categorisation of addiction levels was performed using a quartile classification similar to the approach used by Ruiz-Juan and Zarauz (2012): minimum ( $\leq 4.37$ ), low (4.37-4.99), high (5.00-5.62) and maximum $(\geq 5.62)$ risk of addiction.

\section{Discussion}

All the section was modified considering all the recommendations and rewriting the discussion according to the new results.

Table 4 Crosstab analysis from combining usual distance and levels of addiction risk (chi squared, $\mathrm{X}^{2}$, and significance level)

\begin{tabular}{|c|c|c|c|c|c|c|c|c|}
\hline & \multicolumn{2}{|c|}{$10 \mathrm{~km}$} & \multicolumn{2}{|l|}{$21 \mathrm{~km}$} & \multicolumn{2}{|c|}{$42 \mathrm{~km}$} & \multicolumn{2}{|l|}{ Total } \\
\hline & $\mathrm{X}^{2}$ & $\mathrm{p}$ & $\mathrm{X}^{2}$ & $\mathrm{p}$ & $\mathrm{X}^{2}$ & $\mathrm{p}$ & $\mathrm{X}^{2}$ & $\mathrm{p}$ \\
\hline Sex & 2.12 & .546 & 1.89 & .594 & 1.63 & .652 & 1.80 & .614 \\
\hline Age & 8.27 & .218 & 5.44 & .488 & 3.26 & .352 & 11.33 & .078 \\
\hline Club-membership & 8.40 & $.038^{*}$ & .314 & .957 & 3.39 & .335 & 11.89 & $.008 * *$ \\
\hline Civil Status & 7.73 & .562 & 7.47 & .588 & 1.93 & .993 & 8.30 & .503 \\
\hline Parenthood & 3.05 & .384 & 3.25 & .355 & 3.88 & .274 & 2.98 & .394 \\
\hline Employment Situation & 10.40 & .319 & 6.87 & .650 & 5.74 & .765 & 11.54 & .240 \\
\hline Running expertise years & 9.81 & .133 & 7.50 & .277 & 3.15 & .789 & 14.09 & $.029 *$ \\
\hline Duration of training session & 19.81 & $.003 *$ & 16.56 & $.011 * *$ & 4.28 & .638 & 28.68 & $.000 * * *$ \\
\hline $\mathrm{Km} /$ week & 29.93 & $.008 * *$ & 14.72 & .099 & 9.43 & .396 & 41.77 & $.000 * * *$ \\
\hline Training frequency/week & 28.02 & $.000 * * *$ & 17.61 & $.007 * *$ & 13.65 & $.034 *$ & 61.18 & $.000 * * *$ \\
\hline Date of first race & 6.25 & .181 & 8.29 & .218 & 4.47 & .613 & 6.69 & .350 \\
\hline Distance of first race & 7.07 & .314 & 1.60 & .657 & 5.38 & .496 & 11.74 & .228 \\
\hline Best $10 \mathrm{~km}$ & 9.59 & .384 & 7.55 & .272 & 7.45 & .589 & 21.31 & $.011 *$ \\
\hline Best $21 \mathrm{~km}$ & 17.46 & $.042 *$ & 2.46 & .982 & 8.43 & .491 & 19.76 & $.019 *$ \\
\hline Best 42 km & 11.56 & .239 & 10.52 & .309 & 12.56 & .183 & 22.38 & $.008 * *$ \\
\hline Additional Gym Attendance & 11.23 & $.011 *$ & 2.82 & .419 & 4.52 & .210 & 6.90 & .075 \\
\hline
\end{tabular}




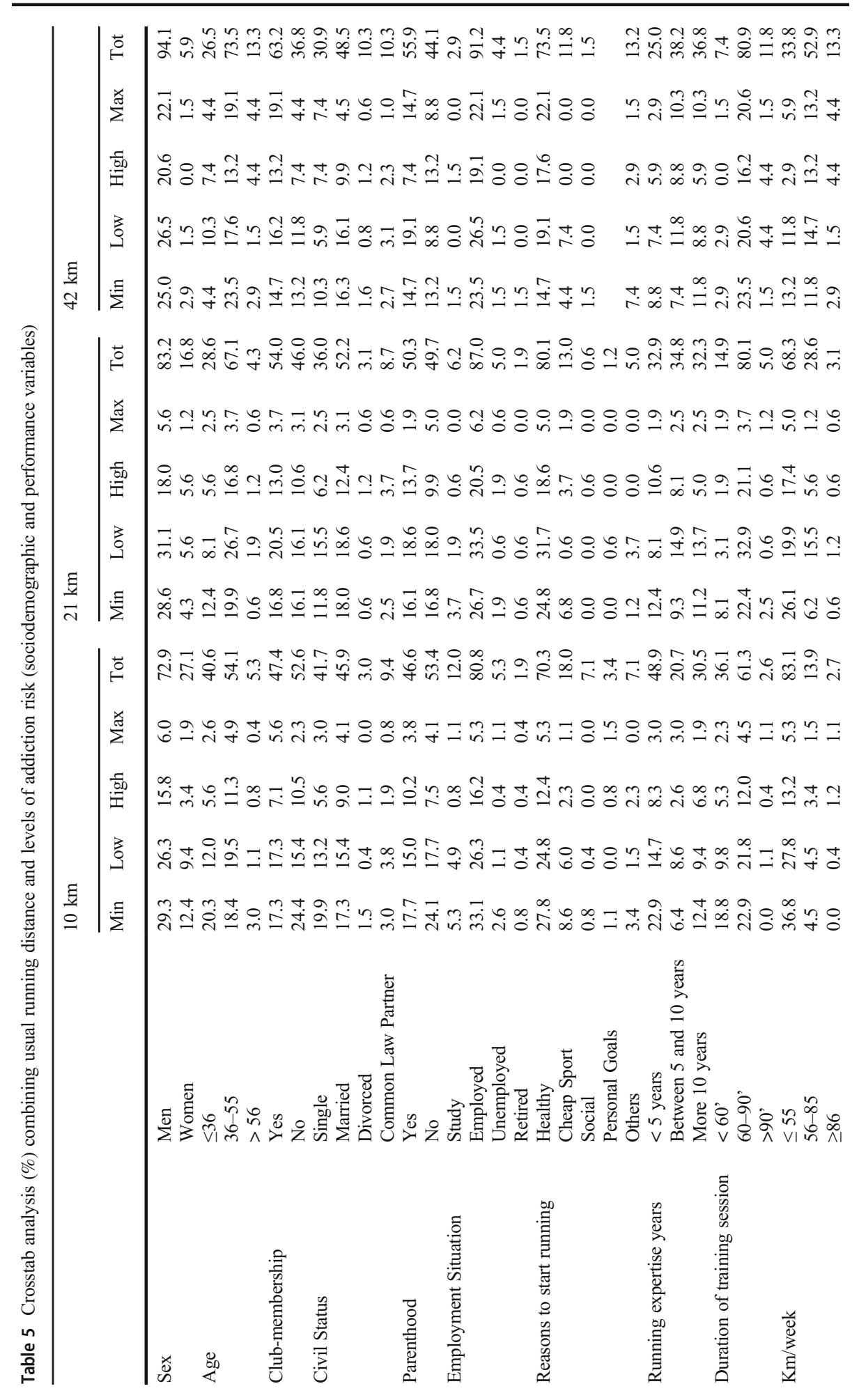




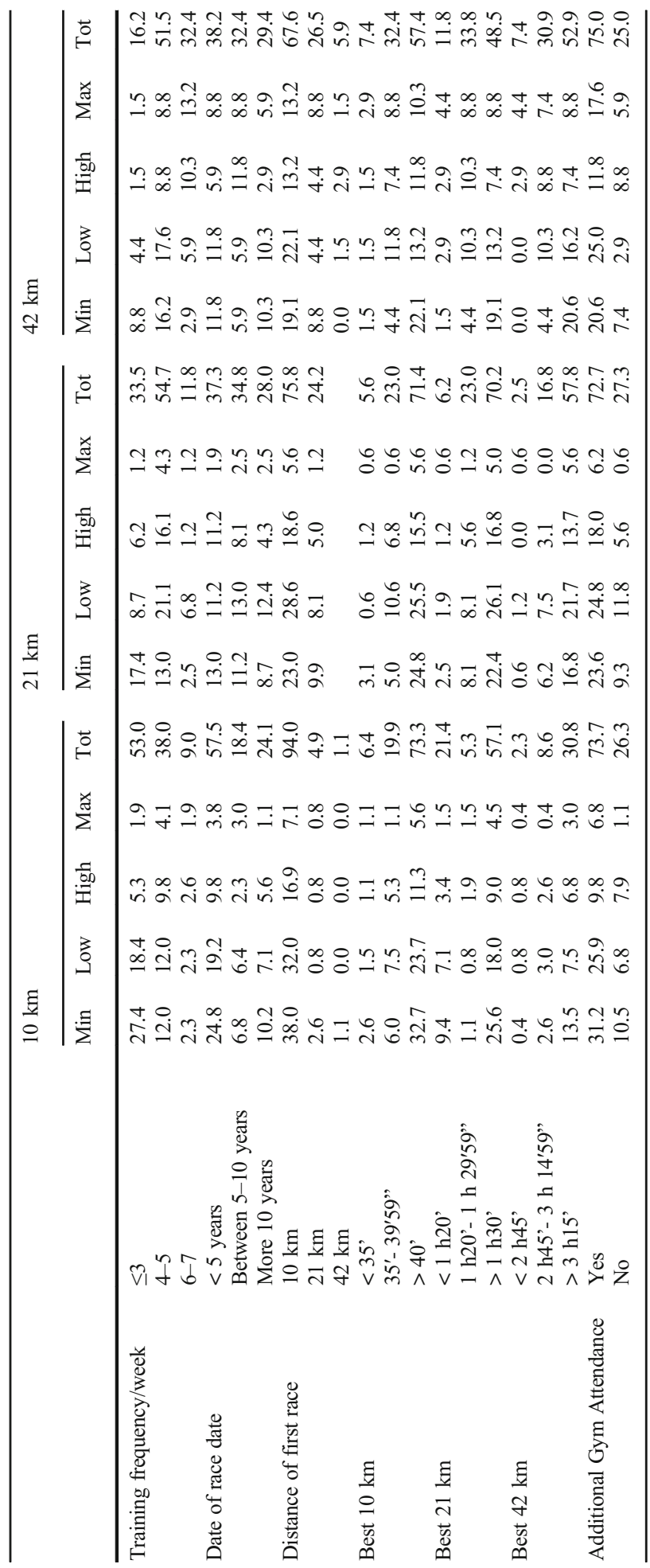


A paragraph of limitations was included.

A paragraph of practical implication was included at the end of the paper.

\section{Conclusions}

The conclusions section was completely modified to reflect the new results.

Section updated by deleting unused references and adding new ones included in the document.

Publisher's Note Springer Nature remains neutral with regard to jurisdictional claims in published maps and institutional affiliations. 